\title{
Seasonal cycle of inbreeding and recombination of the parasitic mite Varroa destructor in honeybee colonies and its implications for the selection of acaricide resistance
}

Alexis L. Beaurepaire ${ }^{1,2}$, Klemens J. Krieger ${ }^{3}$ and Robin F.A. Moritz ${ }^{1,4}$

1) Institut für Biologie, Martin-Luther-University Halle-Wittenberg, Halle a.d. Saale. Germany

2) UR 406 Abeilles et Environnement, INRA, Centre de Recherche Provence-AlpesCôte d'Azur, Avignon, France

3) Bayer Animal Health GmbH, Leverkusen

4) Dept Zoology and Entomology University of Pretoria, Pretoria, South Africa

\section{Corresponding author:}

Alexis Louis Beaurepaire

UR 406 Abeilles et Environnement

INRA, Centre de Recherche Provence-Alpes-Côte d'Azur

Avignon

France

Email: Alexis.Beaurepaire@inra.fr 


\section{Highlights}

- We studied the temporal evolution of $V$. destructor dynamics and genetic structure

- Our predictions show that the mite performs only inbreeding in the early infection stage

- As the mite population grows, multiple infestation and admixture become frequent

- We confirmed this model with data showing increased recombination over a season

- This two-phase dynamics has important significance for acaricide resistance 
Graphical abstract

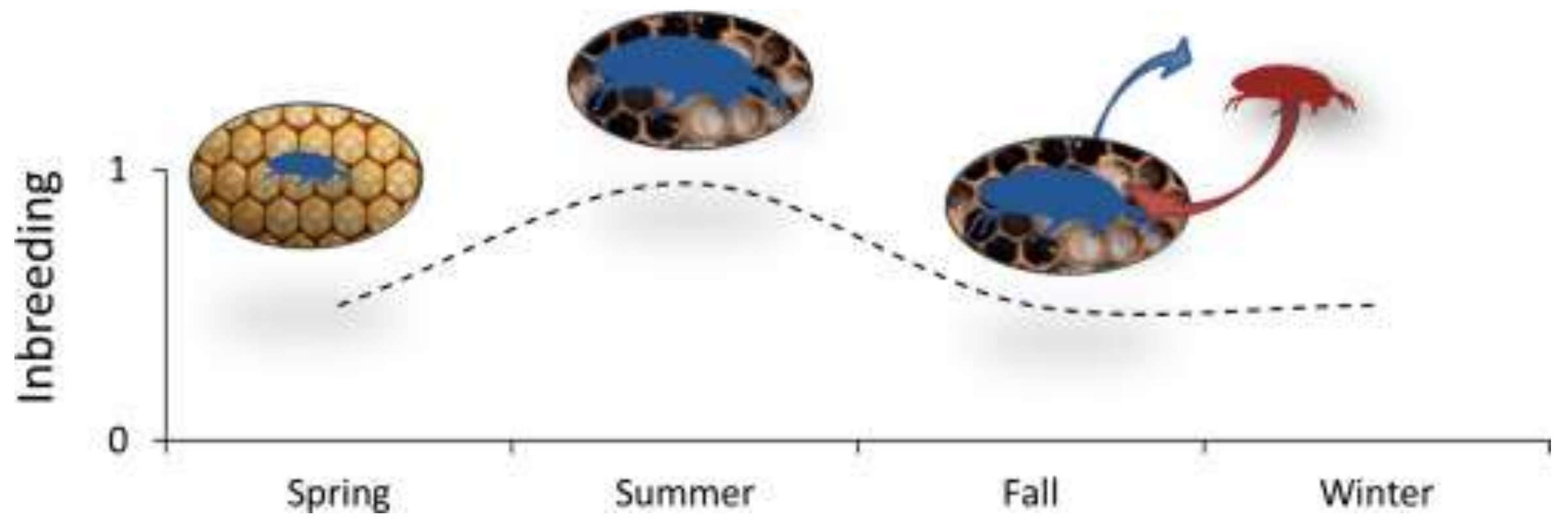




\begin{abstract}
Varroa destructor is the most devastating parasite of the Western honeybee, Apis mellifera. In the light of the arm race opposing the host and its parasite, the population dynamics and genetic diversity of these organisms are key parameters. However, the life cycle of $V$. destructor is characterized by extreme inbreeding due to full sibling mating in the host brood cells. We here present an equation reflecting the evolution of inbreeding in such a clonal system, and compare our predictions with empirical data based on the analysis of seven microsatellite markers. This comparison revealed that the mites perform essentially incestuous mating in the beginning of the brood season. However, this pattern changes with the development of mite infestation. Despite the fact that the overall level of genetic diversity of the mites remained low through the season, multiple inbred lineages were identified in the mites we sampled in June. As a response to the decrease of brood availability and the increase of the parasite population in parallel in the colonies, these lineages recombined towards the end of the season as mites co-infest brood cells. Our results suggest that the ratio of the number of mite per brood cell in the colony determines the genetic structure of the populations of $V$. destructor. This intracolonial population dynamics has great relevance for the selection of acaricide resistance in $V$. destructor. If chemical treatments occur before the recombination phase, inbreeding will greatly enhance the fixation of resistance alleles at the colony level.
\end{abstract}

Keywords: Population Genetics / Host-Parasite Coevolution / Population dynamics / Microsatellites / Varroa destructor / Apis mellifera 


\section{Introduction}

The Western honeybee (Apis mellifera) has been introduced in Asia for honey and bee product business for over a century (Moritz et al., 2005). Due to these introductions, A. mellifera came in contact with a broad range of Asian honeybees' parasites and pathogens, including the Varroa destructor mite. This ectoparasite successfully established in its new host and consequently spread worldwide due to transhumance (Rosenkranz et al., 2010). Today, $V$. destructor represents a major threat for this ecologically and economically important species (Le Conte et al., 2010). On the first hand, the parasite feeds on the haemolymph of honeybee brood and adults, weakening these individuals. But the most devastating effect of the mite comes from the role it plays as a vector for multiple viruses (Genersch and Aubert, 2010; Martin, 2001; Wilfert et al., 2016). This parasite is so devastating that if colonies are not treated, they succumb within 2-3 years (Fries et al., 2006).

As for all invasive species, knowledge on the population dynamics and population genetics are essential to understand the invasion process of $V$. destructor and help establishing effective management measures (Sakai et al., 2001). Among other factors, the population structure and genetic diversity of the introduced population will greatly influence the outcome of the invasion as it will influence the susceptibility to inbreeding depression (Ellstrand et al., 1993; Nieminen et al., 2001) and determine the potential for the emergence and spread of adaptations to the novel environment (Fisher 1930; Sax et al., 2007). These parameters are even more important in parasites like $V$. destructor, as they can influence the arm race with its host (Carius et al., 2001; Van Valen 1973) and its potential to become resistant to acaricides (Georghiou and Taylor, 1977; Roush and McKenzie, 1987).

Mitochondrial and microsatellite DNA studies revealed almost no genetic variation within the two main $V$. destructor haplotypes that switched host and now infest A. mellifera 
colonies worldwide (Anderson and Trueman, 2000; Solignac et al., 2005). This almost clonal population structure was attributed to the peculiar life cycle of the mite which includes mating of siblings in the sealed brood cell of the honeybee. Typically, a Varroa female enters a honeybee brood cell just before capping and activates its ovaries after a first blood meal on the larva. Then she first produces a single haploid male which will later mate with a series of diploid full-sister female offspring in the cell. Sometimes, more than one mite originally invade the cell. In that case, offspring from different mothers may admix (Fuchs and Langenbach, 1989). As soon as the adult bee emerges, the male(s) die(s) and all mated mature females (mother and offspring) can infect new brood cells after a short phase as phoretic mite on adult bees. Hence, this mating biology results in extreme inbreeding with no exchange among lineages in the population as long as a single female mite infests a cell.

As $V$. destructor became a major pest of A. mellifera, various products have been developed in the last decades to control mite infestations. Among these products, pyrethroids have been massively used by beekeepers because they are easy to apply, effective and relatively inexpensive (Rosenkranz et al., 2010). Yet, despite limited genetic diversity and population admixture, several $V$. destructor populations have developed quick resistance to acaricides such as pyrethroids (Milani, 1999). The resistance mechanisms to these compounds have been identified at the molecular level, and recessive point mutations at the sodium channel appears to render the treatments inefficient (González-Cabrera et al., 2016, 2013; Hubert et al., 2014). Interestingly, these studies showed that mites heterozygous at the resistance locus (having both, the resistance " $r$ " and the wild type "+" alleles and therefore called " $r+$ " hereafter) are commonly found in the colonies and that they are highly susceptible to treatments. However, to date nothing is known about how the population dynamics of the mite may influence the emergence and spread of these adaptations at the colony level. 
In order to understand how the interplay between the honeybee colony dynamics and the mating behaviour of the mite can affect the population structure of $V$. destructor, we first show with a very simple equation how inbreeding is expected to vary in $V$. destructor over the honeybee reproduction season. We then discuss how this estimate may vary due to the colony dynamics of the host. To see how inbreeding varies in a real world setting, we compare these theoretical expectations to empirical data. Finally, we show that this temporal dynamics is of great relevance for genetically based resistance towards acaricides.

\section{Material and Methods}

\subsection{Population dynamics}

In order to illustrate the interplay between the population growth of the host and parasite, we first used the model from Calis et al. (1999) to estimate the population dynamics of $A$. mellifera and $V$. destructor under temperate climate (Figure 1). In such an environment, A. mellifera queens typically lay eggs from the beginning of March until the end of October (Calis et al., 1999). In parallel, we chose to track the evolution of the parasite infestation over a year with 50,100, 500 and 1000 individuals to start the $V$. destructor population.

The reproduction of $V$. destructor is directly dependent on the pupation phase of its host, and takes in average 12 days in worker cells and 14 in drones of A. mellifera (Ifantidis, 1983; Martin, 1995, 1994). Every reproductive cycle being separated by a few days of phoretic phase, up to two reproductive cycles per month can be performed by $V$. destructor. As shown in Figure 1, the parasite population growth is greatly affected by the number of individuals starting the infection. Interestingly, the mite outnumbers the colony brood already in September if 1000 mites originally infest the colony. 


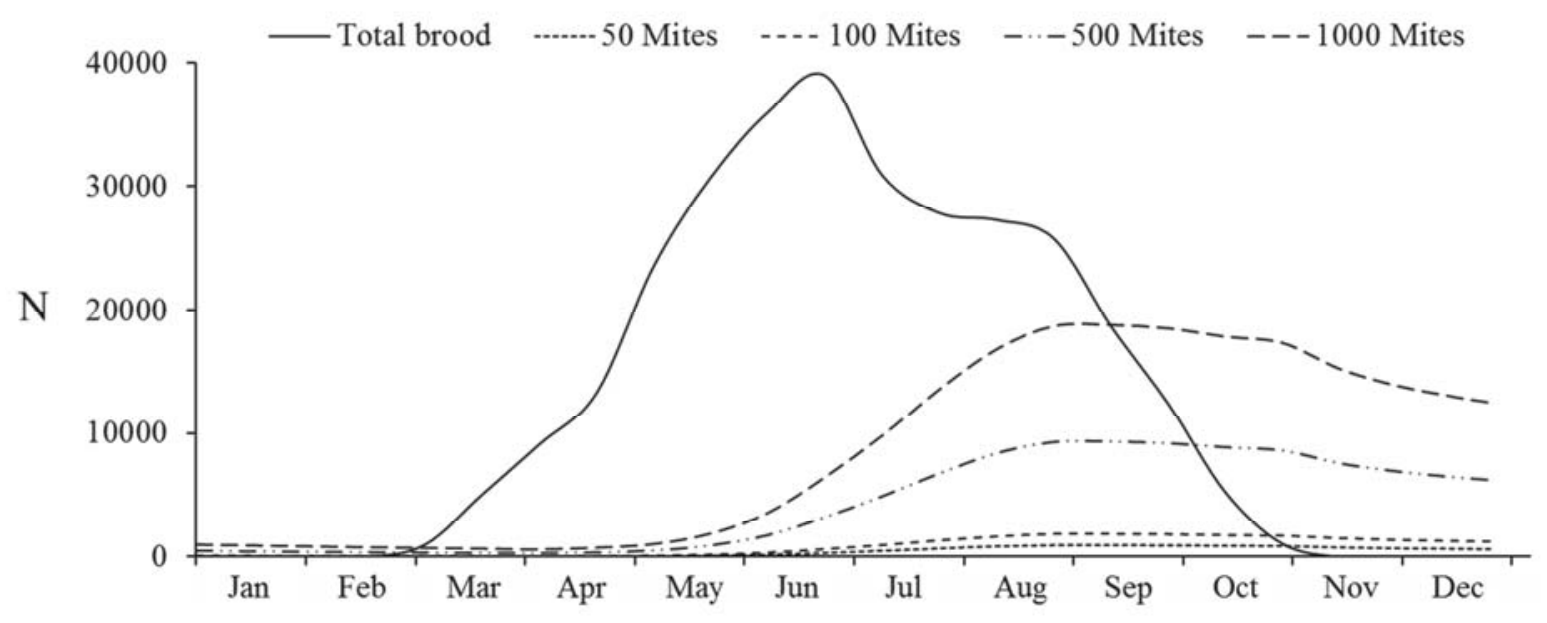

Figure 1 - Honeybee brood and Varroa dynamics

Graph representing the evolution of the amount of A. mellifera brood (solid line; including larvae and pupae of drones and workers), and of V. destructor females (dashed lines) over a year timespan in a temperate climate with a starting population of 50, 100, 500 and 1000 mites. Estimates obtained from the model of Callis et al. (1999). 


\subsection{Evolution of Inbreeding}

To estimate the evolution of the population structure of $V$. destructor under strict incestuous mating, we used a simple equation reflecting the evolution of inbreeding in time. For this estimation, we intentionally neglected the effects of mutation and genetic drift on the genetic structure of Varroa. In fact, the frequency of the former is certainly much reduced in such a short timespan. In addition, as the mite population grows exponentially at the beginning of the brood season due to the large availability of brood cells and only slightly decreases throughout winter (Figure 1), the effects of genetic drift are also very minute. However, the consequences of recombination and migration, which are also not included here, will be discussed in more details in the next parts.

Hence, our estimates of inbreeding results only from recurrent brother-sister mating in the cell. A recurrence equation to determine the inbreeding coefficient $F$ (Wright 1922) in a specific generation $n$ accounting for male haploidy can be derived from Figure S1 as

$$
\begin{aligned}
& F_{n}=0.5^{2}\left(1+F_{n-2}\right)+0.5^{3}\left(1+F_{n-3}\right)+\ldots+0.5^{(n-1)}\left(1+F_{n-(n-1)}\right) \\
& F_{n}=\sum_{x=1}^{n-1}\left(0.5^{(x+1)}\left(1+F_{n-(x+1)}\right)\right)
\end{aligned}
$$

where

$F_{n}=$ inbreeding coefficient in generation $\mathrm{n}(\mathrm{n} \geq 2)$

Figure 2 shows the increase of the probability for two distinct alleles of a given locus to become identical by descent after successive generations of inbreeding. Starting with purely heterozygous individuals $\left(\mathrm{F}_{0}=0\right)$, already more than $75 \%$ of all loci become homozygous after eight generations. As mite reproduction in the honeybee colony is possible throughout the brood season with about 16 mite generations within a year, only very few heterozygote loci will persist under this scenario of recurrent inbreeding. 


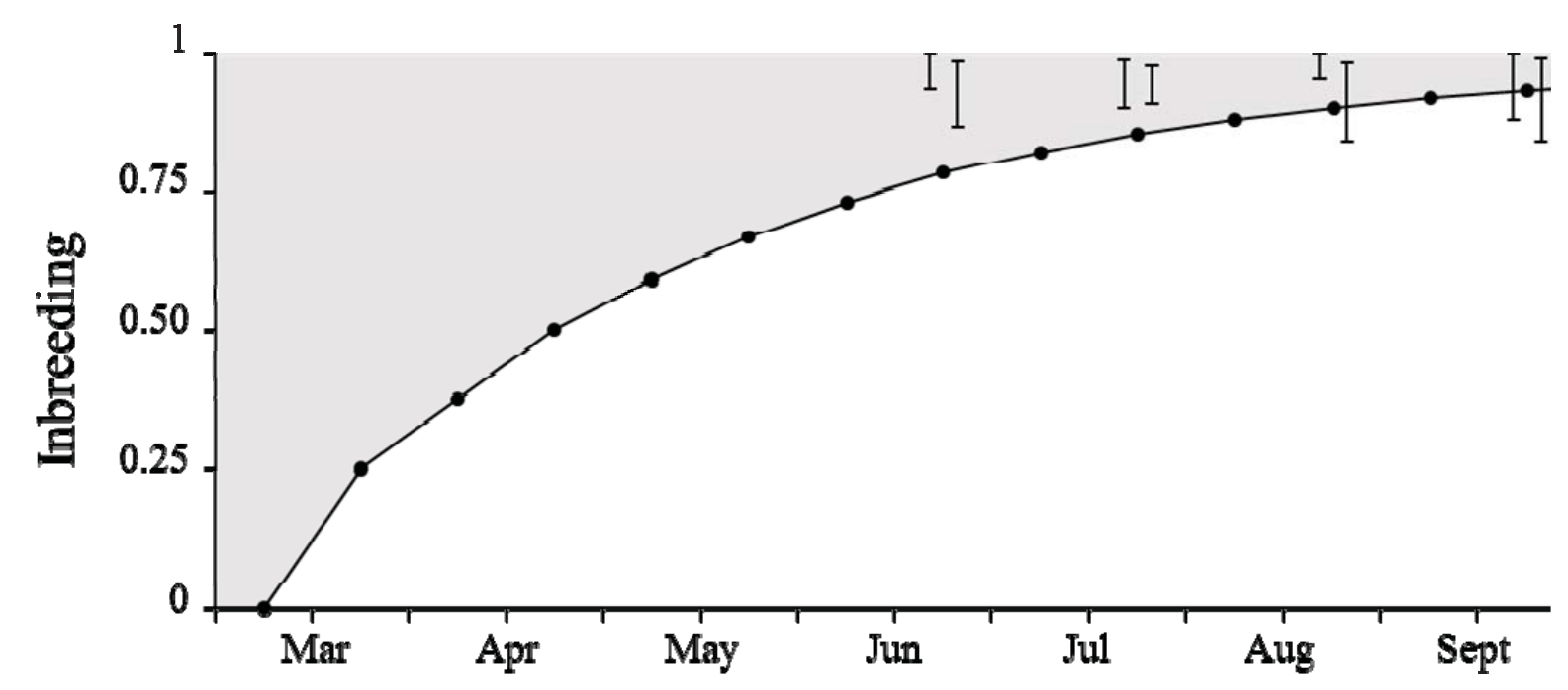

Figure 2 - Inbreeding dynamics in V. destructor

Graph showing the predicted (F; shaded area) and observed (Fest; 95\% CI after 1000 bootstrap over seven loci) inbreeding coefficients in the colonies over the season. The black dots and the curve represent the evolution of inbreeding assuming a most extreme starting situation assuming a non-inbred mite population in early March $(\mathrm{F} 0=0)$. 


\subsection{Interactions between inbreeding and the colony dynamics}

In spite of the life cycle of $V$. destructor with incestuous mating and reproduction in the brood cell, the mites will not always perform inbreeding. If the number of brood cells available for reproduction is smaller than the number of mites in the colony, mites need to coinfect a cell for reproduction (Figure 1). Yet, multiple brood cell infestations may occur even before that stage is reached. Figure 3 shows the temporal changes in single versus multiple cell infestation assuming a random choice of brood cell by $V$. destructor (Fuchs and Langenbach, 1989) based on a Poisson distribution. This figure shows that A. mellifera brood cells will be mainly infested by a single mite as long as the parasite population remains low. However, when the infestation has built up in the colony, the cells will rapidly become saturated with Varroa and the frequency of multiple infestations will grow rapidly.

Thus, the probability of inbreeding in $V$. destructor will depend on the mite-brood ratio and whether multiple females or a single mite infest a brood cell. In the latter case, outbreeding may occur if offspring from two genetically distinct foundresses admix, resulting in an increase in heterozygosity in the mite population. Since colonies typically have large brood nests but only few mites in early spring (Figure 1), multiple infections should be rare and inbreeding will be inevitable if the mites randomly infect brood cells (Figure 3). In autumn, the situation is exactly opposite: there are many mites in the colony but the brood nest is small (Martin, 1998). It is now very likely that mites multiply infest brood cells and heterozygosity may be largely restored.

\subsection{Colony samples}

In order to compare our predictions and estimate the magnitude of the influence of $A$. mellifera colonies dynamics on the population structure of Varroa, we compared the results 


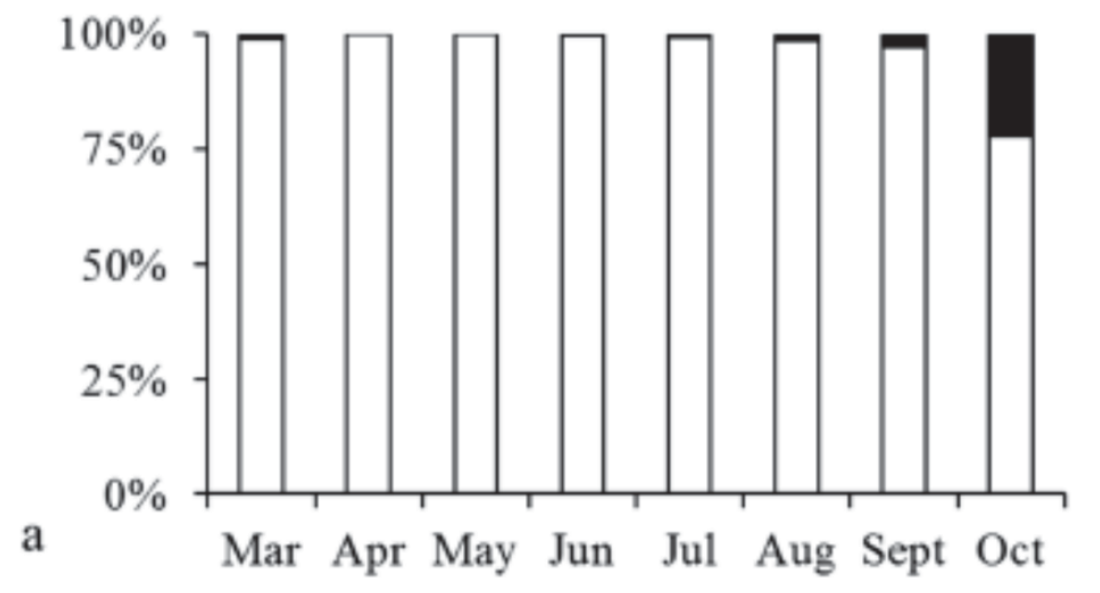

b

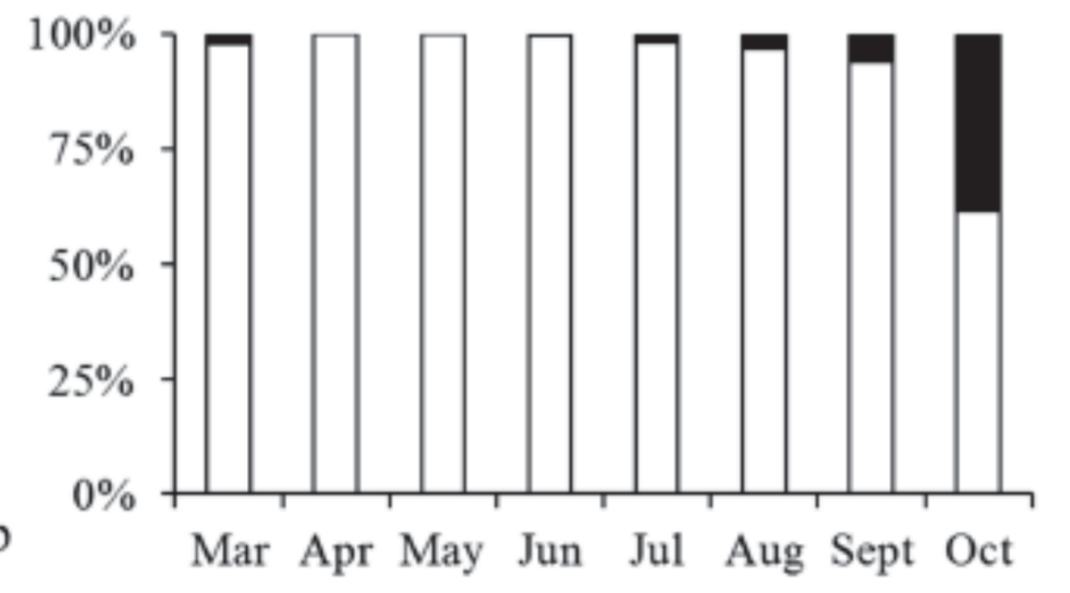

C
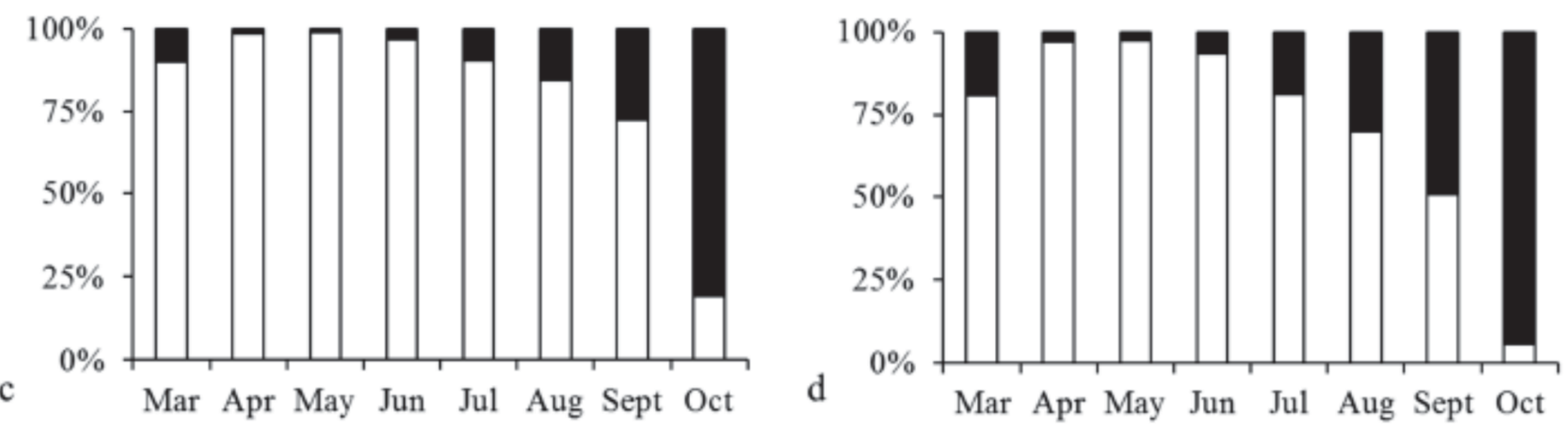

Figure 3 - Temporal evolution of cell infestation in V. destructor

Bar chart representing temporal evolution of the proportions of single (white bars) versus multiple (black bars) infested cells in a colony of A. mellifera on a temperate climate. The likelihood of having one foundress or two foundresses was calculated based on a Poisson distribution with $\lambda$ being the ratio of mite per cells. The different graphs represent starting populations of 50 (a), 100 (b), 500 (c) and 1000 (d) individuals. 


\section{Table 1 - Information on the samples}

Summary of the $V$. destructor sampling, different genetic diversity coefficient estimated over the seven loci and number of lineages sampled in the two honeybee colonies. Time: month of sampling, Inf: infection level (based on how many cells were opened in order to find 12 infected brood), $\mathbf{N}$ : number of mites sampled, $\mathbf{N} /$ cells: average number of mite per cell (N/12; *: the DNA extraction did not work for one individual in this group, therefore only eleven individuals were included in the analyses), $\mathbf{N}_{\mathrm{A}}$ : average number of allelles, $\mathbf{H e}$ : heterozygosity over loci, \%Het: percentage of heterozygous mites (at least one locus), Total: total number of lineages detected, Hom: number of homozygous lineages, Het: number of heterozygous lineages, Rec: number of recombinant lineages, NSE: Non Sampling Error based on the total number of lineages collected.

\begin{tabular}{|c|c|c|c|c|c|c|c|c|c|c|c|c|}
\hline & \multirow{2}{*}{ Time } & \multirow{2}{*}{ Inf } & \multirow{2}{*}{$\mathbf{N}$} & \multirow{2}{*}{ N/cell } & \multirow{2}{*}{$\mathbf{N}_{\mathbf{A}}$} & \multirow{2}{*}{$\mathrm{He}$} & \multirow{2}{*}{$\%$ Het } & \multicolumn{5}{|c|}{ N Lineages } \\
\hline & & & & & & & & Total & Hom & Het & Rec & NSE \\
\hline \multirow{5}{*}{ 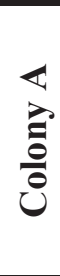 } & June & 0.30 & 13 & 1.08 & 1.57 & 0.022 & 0.15 & 9 & 7 & 2 & 0 & 1.91 \\
\hline & July & 0.34 & 22 & 1.83 & 1.71 & 0.045 & 0.27 & 12 & 6 & 5 & 1 & 2.73 \\
\hline & August & 0.40 & 21 & 1.75 & 1.86 & 0.054 & 0.29 & 13 & 7 & 3 & 3 & 3.30 \\
\hline & September & 0.20 & 16 & 1.33 & 1.86 & 0.107 & 0.44 & 13 & 6 & 5 & 2 & 4.09 \\
\hline & Total & - & 72 & - & 2.29 & 0.057 & 0.29 & 30 & 12 & 13 & 5 & 7.42 \\
\hline \multirow{5}{*}{$\frac{\infty}{\partial}$} & June & 0.30 & 11 & $1.00 *$ & 1.86 & 0.079 & 0.45 & 9 & 5 & 3 & 1 & 2.56 \\
\hline & July & 0.27 & 18 & 1.50 & 1.86 & 0.134 & 0.56 & 13 & 6 & 4 & 3 & 2.87 \\
\hline & August & 0.30 & 13 & 1.08 & 1.71 & 0.120 & 0.62 & 12 & 5 & 4 & 3 & 2.60 \\
\hline & September & 0.53 & 33 & 2.75 & 2.14 & 0.173 & 0.70 & 20 & 4 & 6 & 10 & 1.39 \\
\hline & Total & - & 75 & - & 2.14 & 0.142 & 0.61 & 42 & 13 & 15 & 14 & 6.00 \\
\hline
\end{tabular}


of our equation with empirical data. A total of 147 female adults $V$. destructor were sampled from worker and drone cells in two A. mellifera colonies located in the apiary of the Zoology group of the Martin-Luther University in Halle (Saale), Germany. The hives were not treated with any acaricide throughout the year. The mites were collected in monthly intervals starting in the middle of the parasite infestation peak in June until the end of September 2015 (Table 1). Sealed brood cells were opened until twelve infected cells had been found. The total number of opened cells was recorded to allow for estimates of the brood infection level of the colonies ( $\left.\mathrm{N}_{\text {infected cells }} / \mathrm{N}_{\text {opened cells }}\right)$. Only pigmented mites (mature females) from early stage pupae were removed from the infected cells and stored in $99 \%$ ethanol in order to insure that any multiply infested cells contained different foundresses and a mother and its offspring.

\subsection{Genotyping}

Before DNA isolation, the mites were washed twice with double-distilled water, dried and directly crushed in a PCR plate containing $100 \mu 15 \%$ Chelex solution. $5 \mu 1$ of proteinase $\mathrm{K}$ was then added in each sample. Total mite DNA was isolated from the individuals using standard Chelex thermocycling conditions (Walsh et al., 1991). Four novel polymorphic microsatellite markers (Vdes01-04, Table 2) were designed from the published genome of $V$. destructor (Cornman et al., 2010) using the web software Websat (Martins et al. 2009). In addition, three previously published loci (VJ292 and VJ294 from Solignac et al. (2005) and VD307 from Cornman et al. (2010)) were included in the analyses. All mites were genotyped at these seven microsatellite markers in a MEGABACE DNA Analysis System (GE Healthcare Life Science, Buckinghamshire, England) using the Fragment Profiler software V. 1.2. Hardy-Weinberg tests were performed for each marker, population and over all samples using Fstat V. 2.9.3 (Goudet, 1995). Pairwise linkage disequilibria were estimated for all loci 
Table 2 - Information on the newly designed microsatellite primers

Novel $V$. destructor primers designed for this study. $\mathbf{T}_{\mathbf{A}}$ : annealing temperature of the primer, Size: size of the most frequent allele.

\begin{tabular}{cccccc}
\hline Name & Forward & Reverse & $\mathbf{T}_{\mathbf{A}}$ & Size & Repeat motif \\
\hline Vdes-01 & CGATtTCTGCTCCGTCAGTC & TGTCCTACCAATCATCACCGTA & 60 & 400 & $(\mathrm{TTA})_{12} \ldots(\mathrm{AT})_{7}$ \\
Vdes-02 & TTCAATCAGACCTCCACCTTTT & GCTTATGTAGCCTTTGTCCCAG & 60 & 296 & $(\mathrm{TAA})_{6} \ldots(\mathrm{TA})_{9}$ \\
Vdes-03 & ATATACAGCGCATCGCCTAAA & CGAATAACGCGACAGTTTTGTA & 60 & 303 & $(\mathrm{AT})_{8 . .}(\mathrm{TA})_{7}$ \\
Vdes-04 & AGCTCAGACCATACCGATCAAT & CAACTGTATCATCATCGCCATC & 60 & 272 & $(\mathrm{TA})_{11}$ \\
\hline
\end{tabular}


using the same software and corrections for multiple tests were applied according to De Meeûs (2014).

\subsection{Analysis of the evolution of the population structure of $V$. destructor}

The number and frequency of alleles and the number and percentage of heterozygous individuals were estimated based on the individual genotypes at the seven microsatellite loci for each colony and month. All the following tests were performed using R V. 3.1.2 (R Development Core Team 2008).

The inbreeding coefficient $F_{\text {est }}$ was measured for each colony as the frequency of homozygote genotypes over all loci in the mite samples and its confidence interval estimated with 1000 bootstraps over the seven loci. Using the value of $F_{\text {est }}$ in June, we estimated the evolution of inbreeding assuming strict incestuous mating using the equation (2) described above. We then compared these estimated values to the values of $F_{\text {est }}$ we measured from July to September in both colonies using paired t-tests.

In the two sampled colonies, no more than two alleles were present for each locus from June to August. Hence based on a most conservative coalescent approach, all alleles could be traced back to a single heterozygous mite lineage ("ancestral mite"). It is extremely unlikely to retain heterozygosity at more than one locus in the set of seven markers after eight generations of inbreeding $(<2.02 \%$ based on a Poisson distribution). Hence individuals which had been heterozygote at more than one locus after eight generations were most likely the result of a recombination event between inbred lineages. We determined the regression of the frequency of these recombinant individuals on the frequency of multiply infested brood cells during the season. 


\section{Results}

No significant linkage disequilibrium was found among the seven markers (Table S1). In contrast, significant deviations from Hardy-Weinberg were obtained for all markers and over all markers and populations (Table S2). This result is not surprising given the inbred mating system of $V$. destructor.

The empirical levels of inbreeding we estimated $\left(F_{\text {est }}\right)$ are well above the estimated values from equation (2) in June (Figure 2). This difference indicates that the assumption used in the theoretical approach $\left(\mathrm{F}_{0}=0\right.$; the first individuals are purely heterozygous) was not verified in the empirical data because the levels of inbreeding in the mite population were already important at the beginning of the season. For the following months, the values of inbreeding estimated with equation (2) based on the results of $F_{\text {est }}$ from June were significantly higher than the measured $F_{\text {est }}$ (paired $t$-tests, $p<0.001$, Figure 4). Notably, this difference increased with time, suggesting that the level of incestuous mating diminished progressively through the summer. In accordance with this finding, a significant temporal increase of the frequency of individual heterozygous for at least one locus was detected in both colonies (linear hypothesis test; $p=0.034$ and $p=0.007$ for colony $\mathrm{A}$ and $\mathrm{B}$ respectively). Altogether, these results reflect genetic admixture among inbred lineages in the two colonies (Table 1). Admixture requires the infestation of cells with more than one mite from different lineages. Indeed, also multiple infestations generally increased over the season (Table 1) and significantly affected the frequency of recombinant individuals in our sample $\left(\mathrm{Y}=0.034 \mathrm{x}+0.087 ; \mathrm{r}^{2}=0.501 ; p<0.05\right.$, Figure 5). In addition to this increase in recombination over time, novel alleles were found in each of the colonies in September ( $12.50 \%$ and $6.06 \%$ of sampled individuals, in colony A and B respectively). 

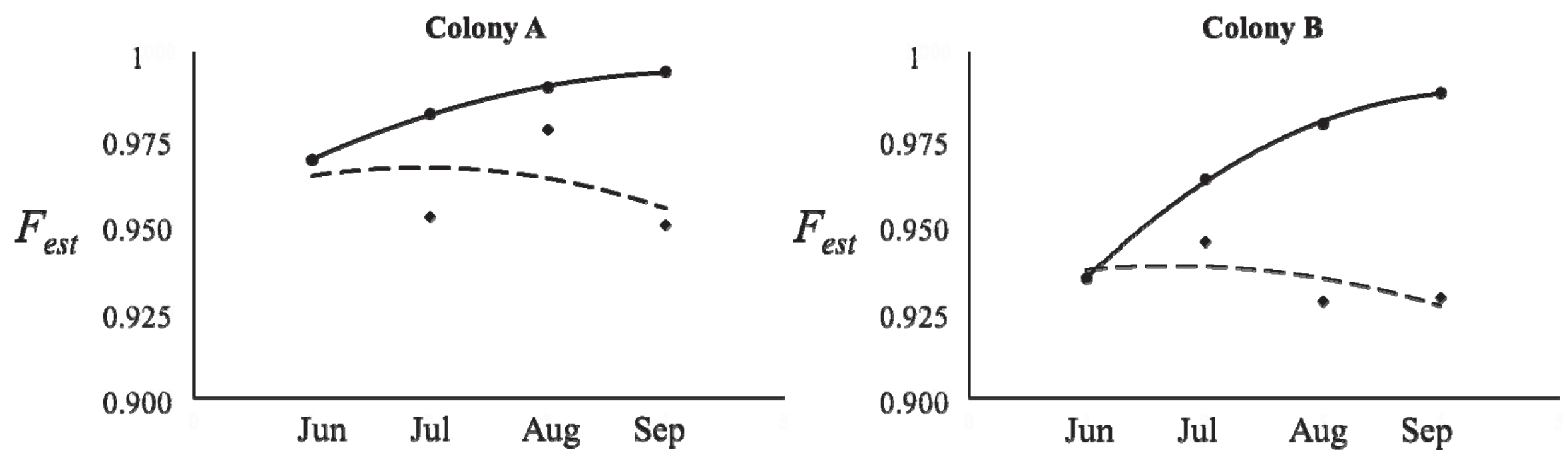

Figure 4 - Figure 4 -Temporal evolution of inbreeding $\left(F_{\text {est }}\right)$

Graph representing the temporal evolution of the inbreeding coefficient (Fest) calculated with the equation (2) (black dots, solid line: polynomial regression curve) and using the genotypes of $\mathrm{V}$. destructor sampled in two A. mellifera colonies (diamonds, dashed line: polynomial regression curve) over four months. 


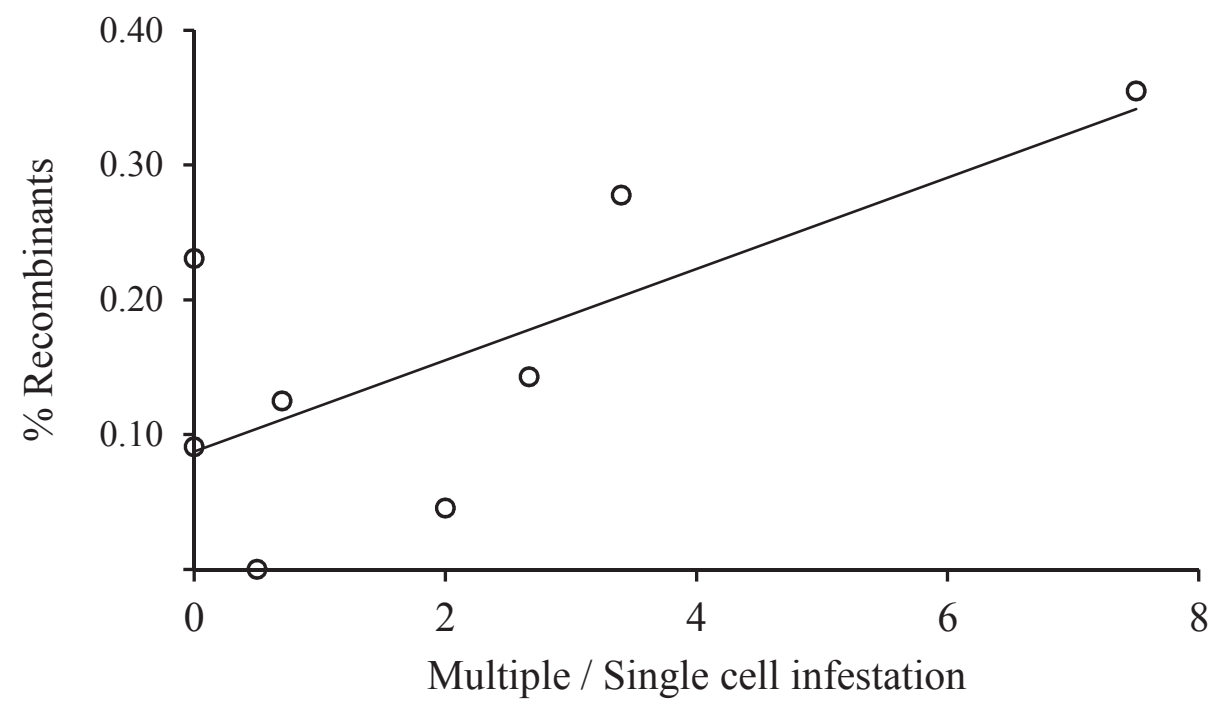

Figure 5 - Relationship between multiple mite infection and the frequency of recombinant genotypes

The plot represents the linear regression of the multiple mite infestation ratio (number of foundresses multiply infecting a brood cell divided by the number of foundresses that infect the brood cells alone) on the estimated frequency of recombinant individuals ( $\mathrm{Y}=$ $\left.0.034 \mathrm{x}+0.087 ; \mathrm{r}^{2}=0.501 ; \mathrm{p}<0.05\right)$ 


\section{Discussion}

Despite the fact that the genetic diversity of the $V$. destructor haplotypes found in $A$. mellifera colonies is considered to be very limited outside of the natural range of the parasite (Solignac et al., 2005), there is growing evidence of significant genetic variation among mites at both the colony and population level (Beaurepaire et al., 2015; Dynes et al., 2016). We here demonstrate that in addition to being genetically diversified, the populations of $V$. destructor are dynamic and complex, as the population structure of this parasite varies temporally in response to the brood availability of its host colony.

As the reproductive season of $V$. destructor typically starts in March in Central Europe when the first eggs are laid by the honeybee queens (Figure 1), approximately four months (or eight generations) may have separated the mites we sampled in June from the first individuals reproducing in the colonies. After eight generations of inbreeding, at least $78.39 \%$ of the alleles would have become identical by descent. The samples from our case study colonies were highly inbred after the mite populations had expanded to elevated levels of infestation early in the season (Figure 2). The mites had apparently mostly infested brood cells alone and their offspring had to reproduce via incestuous mating. This is an inevitable process as long as the mite population is much smaller than the number of brood cells available in the colony, as is typical in the early season but also later if the infestation levels are low. However, despite this extreme inbreeding under large brood nest conditions, genetic drift does not eliminate the initial genetic variation in the mite populations because the number of mites can exponentially expand with every generation. Hence, the population retains all alleles in the colony although within every inbred lineage heterozygosity is almost completely lost and heterozygous loci will become extremely rare. Thus, with incestuous 
mating the mite population in the colony will eventually be comprised of numerous inbred lineages harbouring different alleles all in homozygous state.

However, as the amount of available brood cell decreases and the mite population increases during summer and fall, many of these alleles will recombine because mites inevitably need to co-infest cells for reproduction. In addition, the mite population is not completely closed. Particularly towards the end of the season, there is a considerable influx of foreign mites into the colony (Frey and Rosenkranz, 2014; Greatti et al., 1992) which is supported by enhanced worker drifting that has been suggested to be induced by the mites themselves (Forfert et al., 2015; Kralj and Fuchs, 2006). Admixture of mite populations from different colonies will then take place, increasing the levels of heterozygosity beyond the plain recombination of the resident inbred mite lineages. Also in our study, novel mite genotypes appeared in September suggesting the introduction of new mite lineages into the tested colonies. This might have been caused by the drifting of workers carrying new parasite genotypes from surrounding colonies, although we cannot exclude that we did not detect these alleles earlier on because of the finite sample size of this study.

Clearly our estimates on the evolution of inbreeding in $V$. destructor over time are most conservative, as we chose to start with a single outbred lineage originally in the colonies at the beginning of the season. However, existing inbreeding from the previous year may have been retained over the winter phase. Indeed, our empirical data suggests that multiple mite lineages were present in the colonies at the beginning of the season and that these lineages had substantial levels of inbreeding. Thus, it is not surprising to see the empirically estimated inbreeding coefficients based on the frequency of homozygote loci $\left(F_{\text {est }}\right)$ in June to be higher than the theoretically predicted inbreeding coefficient. Moreover, our equation (2) only addresses the increase of homozygosity within an inbred mite lineage and does not incorporate any admixture of the mite lineages and flux of new alleles in the colony due to 
multiple cell infestation and migration. Hence the lower $F_{\text {est }}$ estimates later in the season exactly reflect recombination of resident inbred lineages the one hand and admixture with novel genotypes on the other.

Even though we screened for microsatellite markers that reflect the random processes of drift and inbreeding, our findings are also most relevant for understanding how selection operates in $V$. destructor. Previous studies have reported that multiple resistant alleles can be found in a given population and that $r r$ individuals (i.e. homozygous with a resistance allele) can resist to acaricide treatment (González-Cabrera et al., 2016, 2013). Interestingly, these mutations were found in $45 \%$ of mites sampled in colonies that were not treated with taufluvalinate, but in $98 \%$ of the treated hives of the same apiaries (González-Cabrera et al., 2016). Our results help understanding how this resistance can take place in $V$. destructor populations infecting $A$. mellifera colonies. Inbreeding will randomly affect all loci including those with recessive alleles for acaricide resistance. So, whereas random mating would result in many $r+$ heterozygotes that are susceptible to the acaricide, incestuous mating will enhance the frequency of $r r$ mites. Increased inbreeding will therefore result in more resistant mites in the colony even before any selection caused by acaricide treatment is applied. Furthermore, acaricide treatments during periods of highly inbred mite populations will accelerate the selection for any recessive resistance allele in the colony. In contrast, treatment during periods of high mite infestation may be more effective. As the ratio of mite per brood cell increases, multiple infestations will become more frequent and will enhance the recombination of inbred lineages, increasing the frequency of susceptible $r+$ individuals in the colony. Thus, if no treatments are performed beforehand, much less mites should survive the treatment after this recombination phase.

To conclude, it may be prudent to consider the interaction between brood availability and inbreeding when designing acaricide application schemes in apicultural management. 
General recommendations for Varroa control are to treat with different compounds throughout the year. However, if these treatments are performed once inbreeding is at its peak, selection of resistance will be swift. In addition, rotating compounds not only in time but also in space between colonies of a given apiary may help preventing the development of acaricide resistance as drift of mites from one colony to another in the late season will enhance the production of heterozygous sensitive individuals.

\section{Acknowledgments}

We would like to thank Robert J. Paxton for allowing us to sample mites in the honeybee colonies based in his apiary (and for sacrificing them for the sake of science), and Kerstin Gössel, Anja Tehel, Rebecca Schwarz, James Ellis and Christina Sann for their help with the sampling. We are also grateful to Petra Liebe and Denise Kleber for their help and support with the lab work. This research was financially supported by Bayer AG. 


\section{References}

Anderson, D.L., Trueman, J.W.H., 2000. Varroa jacobsoni (Acari: Varroidae) is more than one species. Exp. Appl. Acarol. doi:10.1023/A:1006456720416

Beaurepaire, A.L., Truong, T.A., Fajardo, A.C., Dinh, T.Q., Cervancia, C., Moritz, R.F.A., 2015. Host specificity in the honeybee parasitic mite, Varroa spp. in Apis mellifera and Apis cerana. PLoS One 10. doi:10.1371/journal.pone.0135103

Calis, J.N.M., Fries, I., Ryrie, S.C., 1999. Population modelling of Varroa jacobsoni Oud. Apidologie 30, 111-124. doi:10.1051/apido:19990203

Carius, H.J., Little, T.J., Ebert, D., 2001. Genetic variation in a host-parasite association: potential for coevolution and frequency-dependent selection. Evolution (N. Y). 55, $1136-1145$.

Cornman, R.S., Schatz, M.C., Johnston, J.S., Chen, Y.-P., Pettis, J., Hunt, G., Bourgeois, L., Elsik, C., Anderson, D., Grozinger, C.M., Evans, J.D., 2010. Genomic survey of the ectoparasitic mite Varroa destructor, a major pest of the honey bee Apis mellifera. BMC Genomics 11, 602. doi:10.1186/1471-2164-11-602

De MeeÛs, T., 2014. Statistical decision from $\mathrm{k}$ test series with particular focus on population genetics tools: A DIY notice. Infect. Genet. Evol. 22, 91-93. doi:10.1016/j.meegid.2014.01.005

Dynes, T.L., De Roode, J.C., Lyons, J.I., Berry, J.A., Delaplane, K.S., Brosi, B.J., 2016. Fine scale population genetic structure of Varroa destructor, an ectoparasitic mite of the honey bee (Apis mellifera). Apidologie. doi:10.1007/s13592-016-0453-7

Ellstrand, Elam, Elam, D.R., 1993. Population genetic consequences of small population size: implications for plant conservation. Annu. Rev. Ecol. Syst. 24, 217-242.

Forfert, N., Natsopoulou, M.E., Frey, E., Rosenkranz, P., Paxton, R.J., Moritz, R.F.A., 2015. Parasites and pathogens of the honeybee (Apis mellifera) and their influence on intercolonial transmission. PLoS One 10, 1-14. doi:10.1371/journal.pone.0140337

Frey, E., Rosenkranz, P., 2014. Autumn invasion rates of Varroa destructor (Mesostigmata: Varroidae) into honey bee (Hymenoptera: Apidae) colonies and the resulting increase in mite populations. J. Econ. Entomol. 107, 508-515. doi:10.1603/EC13381

Fries, I., Imdorf, A., Rosenkranz, P., 2006. Survival of mite infested (Varroa destructor) honey bee (Apis mellifera) colonies in a Nordic climate. Apidologie 37, 564-570. doi:10.1051/apido

Fuchs, S., Langenbach, K., 1989. Multiple infestation of Apis mellifera L. brood cells and reproduction in Varroa jacobsoni Oud. Apidologie 20, 257-266. doi:10.1051/apido:19890308

Genersch, E., Aubert, M., 2010. Emerging and re-emerging viruses of the honey bee (Apis mellifera L.). Vet. Res. doi:10.1051/vetres/2010027

Georghiou, G.P., Taylor, C.E., 1977. Genetic and biological influences in the evolution of insecticide resistance. J. Econ. Entomol. 70, 319-323.

González-Cabrera, J., Davies, T.G.E., Field, L.M., Kennedy, P.J., Williamson, M.S., 2013. An amino acid substitution (L925V) associated with resistance to pyrethroids in Varroa destructor. PLoS One 8. doi:10.1371/journal.pone.0082941 
González-Cabrera, J., Rodríguez-Vargas, S., Davies, T.G.E., Field, L.M., Schmehl, D., Ellis, J.D., Krieger, K., Williamson, M.S., 2016. Novel Mutations in the Voltage-Gated Sodium Channel of Pyrethroid-Resistant Varroa destructor Populations from the Southeastern USA. PLoS One 11, e0155332. doi:10.1371/journal.pone.0155332

Goudet, J., 1995. FSTAT (Version 1.2): a computer program to calculate F-statistics. Heredity (Edinb). 96, 485-486. doi:10.1017/CBO9781107415324.004

Greatti, M., Milani, N., Nazzi, F., 1992. Reinfestation of an acaricide-treated apiary by Varroa jacobsoni Oud. Exp. Appl. Acarol. doi:10.1007/BF01218569

Hubert, J., Nesvorna, M., Kamler, M., Kopecky, J., Tyl, J., Titera, D., Stara, J., 2014. Point mutations in the sodium channel gene conferring tau-fluvalinate resistance in Varroa destructor. Pest Manag. Sci. 70, 889-894. doi:10.1002/ps.3679

Ifantidis, M.D., 1983. Ontogenesis of the mite Varroa jacbsoni in worker and drone honeybee brood cells. J Apicul Res 22, 200-206. doi:10.1080/00218839.1983.11100588

Kralj, J., Fuchs, S., 2006. Parasitic Varroa destructor mites influence flight duration and homing ability of infested Apis mellifera foragers. Apidologie 37, 577-587. doi:10.1051/apido:2006040

Le Conte, Y., Ellis, M., Ritter, W., 2010. Varroa mites and honey bee health: can Varroa explain part of the colony losses? Apidologie 41, 353-363. doi:10.1051/apido/2010017

Martin, S.J., 2001. The role of Varroa and viral pathogens in the collapse of honeybee colonies: A modelling approach. J. Appl. Ecol. 38, 1082-1093.

Martin, S.J., 1998. A population model for the ectoparasitic mite Varroa jacobsoni in honey bee ( Apis mellifera ) colonies . Ecological Modelling 109 109, 267-281. doi:10.1016/S0304-3800(98)00059-3

Martin, S.J., 1995. Ontogenesis of the mite [i]Varroa jacobsoni[/i] Oud. in drone brood of the honeybee [i]Apis mellifera[/i] L. under natural conditions. Exp. Appl. Acarol. 19, 199_ 210.

Martin, S.J., 1994. Ontogenesis of the mite Varroa jacobsoni Oud. in worker brood of the honeybee Apis mellifera L . under natural conditions. Exp. Appl. Acarol. 18, 87-100.

Milani, N., 1999. The resistance of Varroa jacobsoni Oud. to acaricides. Apidologie 30, 229 234. doi:10.1051/apido:19990211

Moritz, R.F.A., Härtel, S., Neumann, P., 2005. Global invasions of the western honeybee (Apis mellifera) and the consequences for biodiversity. Ecoscience 12, 289-301. doi:10.2980/i1195-6860-12-3-289.1

Nieminen, M., Singer, M.C., Fortelius, W., Scho, K., 2016. Notes and Comments Experimental Confirmation that Inbreeding Depression Increases Extinction Risk in Butterfly Populations 157, 237-244.

Rosenkranz, P., Aumeier, P., Ziegelmann, B., 2010. Biology and control of Varroa destructor. J. Invertebr. Pathol. doi:10.1016/j.jip.2009.07.016

Roush, R.T., McKenzie, J.A., 1987. Insecticide and Acaricide Resistance. Annu. Rev. Entomol. 361-80. doi:10.1016/B978-0-12-374144-8.00144-2

Sakai, A., Allendorf, F., Holt, J., Lodge, M., Molofsky, J., With, K.A., Baughman, S., Cabin, R.J., Cohen, J.E., Ellstrand, N.C., McCauley, D.E., O’Neil, P., Parker, I.M., Thompson, J.N., Weller, S.G., 2001. The population biology of invasive species. Annu. Rev. ... 32, 
305-332. doi:10.1146/annurev.ecolsys.32.081501.114037

Sax, D.F., Stachowicz, J.J., Brown, J.H., Bruno, J.F., Dawson, M.N., Gaines, S.D., Grosberg, R.K., Hastings, A., Holt, R.D., Mayfield, M.M., O’Connor, M.I., Rice, W.R., 2007. Ecological and evolutionary insights from species invasions. Trends Ecol. Evol. doi:10.1016/j.tree.2007.06.009

Solignac, M., Cornuet, J.-M., Vautrin, D., Le Conte, Y., Anderson, D., Evans, J., Cros-Arteil, S., Navajas, M., 2005. The invasive Korea and Japan types of Varroa destructor, ectoparasitic mites of the Western honeybee (Apis mellifera), are two partly isolated clones. Proc. Biol. Sci. 272, 411-419. doi:10.1098/rspb.2004.2853

Walsh, P.S., Metzger, D.A., Higuchi, R., 1991. Chelex 100 as a medium for simple extraction of DNA for PCR-based typing from forensic material. Biotechnique v.10, 506-513. doi:10.2144/000113897

Wilfert, L., Long, G., Leggett, H.C., Schmid-Hempel, P., Butlin, R., Martin, S.J.M., Boots, M., 2016. Deformed wing virus is a recent global epidemic in honeybees driven by Varroa mites. Science (80-. ). 351. doi:10.1126/science.aac9976

Wright, S. 1922. Coefficients of inbreeding and relationship. American Naturalist 56, 330 338 


\section{Supplementary Information}

\section{Table S1 - Results of the linkage disequilibrium test}

Linkage disequilibrium estimated for all pair of markers using Fstat v.2.9.3 (Goudet, 1995). After Bonferroni correction, adjusted $P$-value for 5\% nominal level was 0.002381 .

\begin{tabular}{|c|c|c|}
\hline \multicolumn{2}{|c|}{ Marker pairs } & $p$ \\
\hline 5307 & \& VJ292 & \\
\hline & $\& \mathrm{VJ}^{2}$ & \\
\hline אI: & \& VJ & \\
\hline & $\& \mathrm{Vc}$ & \\
\hline VJ292 & $\& \mathrm{Vd}$ & \\
\hline 294 & $\& \mathrm{Vc}$ & \\
\hline 307 & $\& V$ & \\
\hline $\mathrm{J} 292$ & $\& \mathrm{Vc}$ & \\
\hline J294 & $\& \mathrm{Vc}$ & \\
\hline Vdes-0 & $\& V$ & \\
\hline VD307 & $\& \mathrm{Vc}$ & \\
\hline VJ292 & $\& \mathrm{Vd}$ & \\
\hline VJ294 & $\& \mathrm{Vd}$ & \\
\hline Vdes-0 & $\& \mathrm{Vd}$ & \\
\hline Idor 0 & $\& \mathrm{Vd}$ & \\
\hline VD307 & $\& \mathrm{Vd}$ & \\
\hline 1) & $\& \mathrm{Vd}$ & \\
\hline VJ294 & $\& \mathrm{Vd}$ & \\
\hline$V$ Vac 0 & $\& \mathrm{Vde}$ & \\
\hline & \& Vdes-04 & \\
\hline Vdes-03 & \& Vdes-04 & 0.04048 \\
\hline
\end{tabular}

\section{Table S2 - Results of the Hardy-Weinberg test}

Hardy-Weinberg tests estimated for all pair of markers and over all markers and populations using Fstat v.2.9.3 (Goudet, 1995) using the $\mathrm{F}_{\text {IS }}$ statistics and based on 2000 randomisations. The results shown are the probability that the randomisations are larger than the observed values of $F_{I S}$.

\begin{tabular}{cc}
\hline Marker & Result \\
\hline VD307 & $<0.05$ \\
VJ292 & $<0.05$ \\
VJ294 & $<0.05$ \\
Vdes-01 & $<0.05$ \\
Vdes-02 & $<0.05$ \\
Vdes-03 & $<0.05$ \\
Vdes-04 & $<0.05$ \\
All Loci & $<0.05$ \\
\hline
\end{tabular}




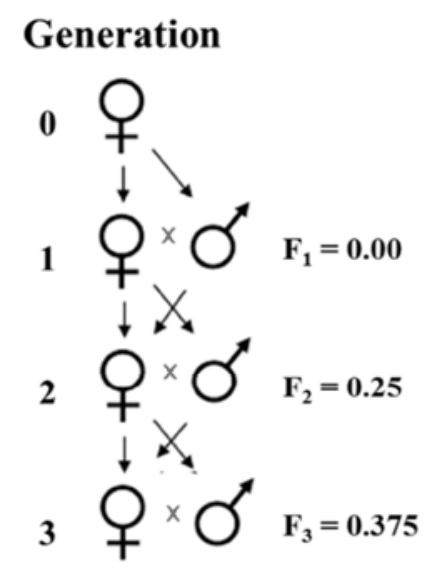

\section{Figure S1 - Inbreeding pedigree of $V$. destructor lineages}

Pedigree showing the first four generations of inbreeding. Females are diploid but males are haploid causing a swift increase in the inbreeding coefficient. The inbreeding coefficient of a female in generation 3 is composed of $F 3=0.5^{2}\left(1+F_{1}\right)+0.5^{3}\left(1+F_{0}\right)=$ $0.375\left(\mathrm{~F}_{0}\right.$ and $\left.\mathrm{F}_{1}=0\right)$. 